\title{
Theoretical Investigations of Vibrational - Rotational Energy of some Hydrogen Halide Molecules
}

\author{
Rafah Abdul Hadi*
}

\begin{abstract}
Date of acceptance 9/6/2007
Abstract:

A theoretical study by using computer model is presented to study the energy characteristics of the vibrational - rotational levels as a function of the vibrational and rotational quantum number, respectively. The calculations were based on the basis of a multilevel model taking into account the non-equilibrium population of the rotational levels. The computational investigation has been performed to examine the vibrationalrotational characteristics of some hydrogen halides chemical laser molecules. This program takes into account the various molecules of chemical lasers such as, Hydrogen Fluoride (HF), Deuterium Fluoride (DF), Hydrogen Chloride $(\mathrm{HCl})$, and Deuterium Chloride $(\mathrm{DCl})$. The practical difficulties associated with this type of lasers suggested that alternative route might be quite useful. Thus particular attention was paid to the development of computer program to investigate various energy characteristics. Comparison of the calculated results for different molecules shows that the highest values for the HF molecule probably due to their higher constants in comparison with the other molecules.
\end{abstract}

\section{Introduction}

The basic idea for creating chemical laser, which consists in direct conversion of the energy released in the process of a chemical reaction into coherent radiant energy, bypassing other forms, goes back to the early 1960's. In chemical lasers, the chemical reactions usually take place between gaseous elements. In this case, the greater part of the reaction's energy is released in the form of the vibrational energy of molecules. Therefore laser transitions are vibrational-rotational, and the wavelengths corresponding to them lie in the range from 1.3 to $26 \mu \mathrm{m}$. The radiation of some wavelengths from this range cannot be obtained using other types of lasers (Ilyushin, 1998).

Laser operation take place via transitions between different energy levels of an atomic or molecular system. But the chemical laser makes use of the only chemical reaction in order to produce the excited atoms or molecules. It is more advantageous to exploit a considerable amount of chemical reaction to generate the excited atoms or molecules, since the chemical laser has a characteristic of considerably high efficiency in energy transformation from chemical energy to light energy (Park and Back, 2005).

Chemical lasers have many attractive features, they produce the highest output power per unite volume and per unite weight. In general, chemical reactions excite vibrational levels and thus the output wavelength is always in the infrared ( $1 \mu \mathrm{m}$ to $12 \mu \mathrm{m})$. If one - shot large power is needed as, for example, in a star wars scenario, chemical lasers can produce large amounts of destructive energy without any electrical power (Das, 1991).In the past, chemical lasers have been used for industrial applications like metal cutting.

*Collage of Science for Women/ University of Baghdad / Physics Department 
However, it's the military application of chemical lasers which has lead to much of the research in this field. A chemical laser can continuously supply megawatt of energy at lightening speed. This unique ability of chemical lasers is used to develop laser weapon technology which would destroy a typical ballistic missile at the speed of light (Palekar, 2004).

A multilevel model have been developed for lasers operation on the basis of the vibrational - rotational transitions in diatomic molecules and taking into account the anharmonicity and nonequilibrium population of the rotational levels. This model was used in a theoretical study of a pulsed chemical $\mathrm{H}_{2}-\mathrm{F}_{2}$ laser (Igoshin and Pichugin,1992). It makes it possible to calculate the rotational parameters for different chemical laser molecules. The use of such a model will make it possible to calculate the energy characteristics of multiline chemical laser radiation, and also to investigate the influence of the rotational constant of the different chemical laser molecules such as HF, $\mathrm{DF}, \mathrm{HCl}$, and $\mathrm{DCl}$ on their characteristics. These molecules possess a rich of close together spectral radiation lines

Due to the anharmonicity of the HF and DF molecules (i.e., the energy differences between adjacent vibrational levels in the irrational manifold are not equal), lasing transitions between the various vibrational levels correspond to different laser wavelengths. This due to decrease in spacing at higher energy levels ( e.g. from level 6 to level 5 than from 1 to level 0)(Deutsch, 1967). Hydrogen fluoride emits in the wavelength region of $2.6-3.3 \mu \mathrm{m}$ in atmosphere absorption band. In substitution of deuterium for $\mathrm{H}$ the wavelength shifts to the region of $3.5-$ $4.5 \mu \mathrm{m}$ with no absorption by atmosphere (Perram, 2000).
To compare $\mathrm{H}_{2}+\mathrm{Cl}_{2}$ with the $\mathrm{H}_{2}+\mathrm{F}_{2}$ systems. The combination of: (i) IR spectral range suitable for molecular vibrations attack as well as for transmittance in atmosphere, (ii) large number of lines, and (iii) high peak power, makes $\mathrm{HCl}$ laser very promising tool (Bravy, 1998).But its short coming, in comparison with HF laser, was a low efficiency, arising from the absence in $\mathrm{H}_{2}+\mathrm{Cl}_{2}$ system of fast chain reaction inherent to $\mathrm{H}_{2}+\mathrm{F}_{2}$ one. The HF pulsed chemical laser on a chain $\mathrm{H}_{2}+\mathrm{F}_{2}$ are indisputable leader among the pulsed lasers. The chemical reaction is initiated for example by $\mathrm{F}$ atoms photolytical formation $\mathrm{F}_{2}+\mathrm{hv} \rightarrow 2 \mathrm{~F}$, followed by fast chain reaction (Detweiler and et al, 2005)

$\mathrm{F}+\mathrm{H}_{2} \rightarrow \mathrm{HF}^{*}+\mathrm{H} \quad \Delta \mathrm{H}=-134 \mathrm{~kJ} / \mathrm{mole}$

$\mathrm{H}+\mathrm{F}_{2} \rightarrow \mathrm{HF}^{*}+\mathrm{F} \quad \Delta \mathrm{H}=-406 \mathrm{~kJ} / \mathrm{mole}$

where vibrationally excited $\mathrm{HF}^{*}$, molecules appear in both links of the chain.

In most respects $\mathrm{HF}$ and $\mathrm{HCl}$ are quite similar; the main difference in the chemistry of the two systems lies in the lower bond energy of $\mathrm{HCl}(4.43 \mathrm{eV})$ while the HF bond energy $(5.86 \mathrm{eV})$ (Eletskii, 1981). Resulting generally in lower reaction exothermicities for $\mathrm{HCl}-$ forming reactions. In particular, in the $\mathrm{H}_{2}-\mathrm{Cl}_{2}$ chain reaction, only the reaction

$\mathrm{H}+\mathrm{Cl}_{2} \rightarrow \mathrm{HCl}^{*}+\mathrm{Cl} \quad \Delta \mathrm{H}=-189 \mathrm{~kJ} / \mathrm{mole} \quad$ (3) leads to vibrational excitation. The reaction

$\mathrm{Cl}+\mathrm{H}_{2} \rightarrow \mathrm{HCl}^{*}+\mathrm{Cl} \quad \Delta \mathrm{H}=+4 \mathrm{~kJ} /$ mole $\quad$ (4) produces only $\mathrm{HCl}$ in the ground state. Thus, although the $\mathrm{H}_{2}-\mathrm{Cl}_{2}$ chain reaction has been used for flash lamp pumped $\mathrm{HCl}$ lasers, the system is inherently inefficient (Stitch, 1979).

It can be shown that $\mathrm{HCl}$ molecules appear in the zero vibrational state $(\mathrm{E} 0=0)$ in the reaction $(4)$, and only $0.015 \%$ of the $\mathrm{HCl}$ molecules formed occupy the $v=6$ level corresponding to an energy of $189 \mathrm{~kJ} / \mathrm{mole}$ (Kondratiev,1969).The small fraction of $\mathrm{HCl}$ molecules carrying away a large 
amount of vibrational energy at the moment of their formation, as well as the high probability of vibrational and rotational energy dissipation by molecular collisions.

Excitation of the vibrational rotational levels of the DF molecules in the chain reaction (Azarov and et al, 2000)

$\mathrm{F}+\mathrm{D}_{2} \rightarrow \mathrm{DF}+\mathrm{D}$

$\mathrm{D}+\mathrm{F}_{2} \rightarrow \mathrm{DF}+\mathrm{F}$

$\Delta \mathrm{H}=-544$

$\mathrm{kJ} / \mathrm{mole}$

In comparison of the heat of reaction $(\Delta \mathrm{H})$ of both $\mathrm{HF}$ and DF the excitation of the vibrational - rotational levels consume approximately the same amounts of the energy released in the chemical reaction (Gross and Bott, 1976). However, the specific output energies available from $\mathrm{HF}$ and $\mathrm{DF}$ pulsed chemical laser based on this chain reaction, usually differ by a factor of $2-2.5$

(Nichols and at el, 1976). Various investigators are of the opinion that this is associated with the larger ( compared with HF) number of the vibrational - rotational levels of the DF molecule (over which the excitation energy is distributed ) and the smaller Einstein coefficients and rate constants of the chain reaction of the DF laser.

The main distinction between DF and HF lasers lies in the fact that during the chain reaction reactions $\mathrm{D}_{2}+\mathrm{F}_{2}$ and $\mathrm{H}_{2}+\mathrm{F}_{2}$ the equal energy of chemical interaction is spent on exciting different numbers $(\mathrm{N})$ of the $\mathrm{DF}$ and $\mathrm{HF}$ molecules. Therefore the gain on the transitions of DF molecule are lower than those of HF molecule, and for excitation of DF laser generation a high quality resonator is required.

\section{Theory}

Theory of any vibrational-rotational state expressed in its wave number equivalent and measured in cm-1 units, can be written to a good approximation as (Basov and et al, 1990)

$E_{v, J}=G(v)+B v J(J+1)-D_{v} J^{2}(J+1)+$ where

$\mathrm{v}=$ the vibrational quantum number $(0$,

1,2 , etc.)

$\mathrm{J}=$ the rotational quantum number $(0,1,2$, etc. $)$

$\mathrm{B}_{\mathrm{v}}=$ rotational constant for vibrational state $\mathrm{V}$

$D_{v}=$ the centrifugal distortion constant for vibrational state $\mathrm{v}$ with

$\mathrm{G}(\mathrm{v})=$ the vibrational energy

$B_{v}=B_{e}-\alpha_{e}(v+1 / 2)+$ (smaller correction terms, expressed in a power series in $(v+1 / 2))$

and

$\mathrm{G}(\mathrm{v})=\omega_{\mathrm{e}}(\mathrm{v}+1 / 2)-\omega_{\mathrm{e}} \chi_{\mathrm{e}}(\mathrm{v}+1 / 2)_{2}+$ (smaller terms)

The rotational energy is given by the following expression

$\mathrm{F}(\mathrm{J}, \mathrm{v})=\mathrm{J}(\mathrm{J}+1)\left[\mathrm{B}_{\mathrm{e}}-\alpha_{\mathrm{e}}(\mathrm{v}+1 / 2)\right]$

where

$\mathrm{B}_{\mathrm{e}}=$ the equilibrium rotational constant

$\alpha_{\mathrm{e}}=$ the vibrational - rotational coupling constant

$\omega_{\mathrm{e}}=$ the fundamental vibrational constant

$\omega_{\mathrm{e}} \chi_{\mathrm{e}}=$ the first anharmonic correction constant.

To have an allowed vibration - rotation absorption transition in a diatomic will all electrons spin - paired, the quantum number ( expressed as $\Delta$ (number) =final value - initial value) must satisfy the following restrictions, called selection rules :

Vibrational quantum number : $\Delta \mathrm{v}=1$ (for the most probable absorption transitions - the "fundamental" transitions) or $\Delta v=2,3,4$, etc., with ever diminishing probability of occurrence (the " overtone" transitions)

rotational quantum number : $\Delta \mathrm{J}= \pm 1$ where those transitions with $\Delta J=-1$ are said to be members of the "P branch" and those with $\Delta \mathrm{J}=+1$ from the "R branch". 


\section{Results and Discussion}

In the present work, the calculations of the vibrational and rotational energy as a function of vibrational and rotational quantum number, respectively for four different hydrogen halides molecules $\mathrm{HF}, \mathrm{DF}, \mathrm{HCl}$ and $\mathrm{DCl}$ are presented by using computer model. The certain Matlab computer model was developed to simulate vibrational rotational energy of these chemical lasers molecules. The model can overpredicted performance of the chain reaction in these types of lasers. These parameters were calculated by using computer modeling based on the values of the equilibrium rotational constant $\left(B_{e}\right)$ which were given for all molecules.

By using equations (2) to calculate the rotational constant for vibrational state $\left(B_{v}\right)$ for four molecules and Figure (2) shows its variation with the vibrational quantum number $\mathrm{v}$. It is seen that $B_{v}$ increases with increasing $v$ for all molecules, particularly at high values of v. It has an initial values for all molecules as one would expected from the equation (2) and the higher value for $\mathrm{HF}$ and the lowest value for DCl. The closest values of $\mathrm{DF}$ and $\mathrm{HCl}$ are due to the small difference between the equilibrium rotational constant of both molecules (for DF $B_{e}=11.00 \mathrm{~cm}^{-1}$ and for $\mathrm{HCl} \mathrm{B} \mathrm{e}_{\mathrm{e}}=10.59 \mathrm{~cm}^{-1}$ ).

The calculations of the vibrational energy are made by using equation (3) and the results of calculation are shown in Figure (3). It can be seen that this factor varies linearly with the vibrational quantum number and the highest value is determined for HF molecule. This is due to the fact the constants of the HF molecule are several orders of magnitude larger than that for the other molecules.

It is quite important to know the rotational energy levels $\mathrm{F}(\mathrm{J}, \mathrm{v})$ in order to predict the population of each vibrational - rotational level. Since the distribution of these level depends on the fraction of energy transferred to the vibrational degree of freedom of a molecule, which is a reaction product. By using the values of $B_{v}$ to calculate the rotational energy (equation 4) as a function of the rotational quantum number at various values of vibrational quantum number $(\mathrm{v})$. The results of the computational series are presented in Figures (4) - (8). Here the rotational energy increases with the rotational quantum number and the highest value is determined for HF molecule as would be expected. The main conclusion that can be drawn on the basis of these figures that there is a small variation in the $F(J, v)$ for different values of $v$ as it can be shown in all figures and the variation approximately in the same manners for all molecules. It means that the rotational energy increases as $\mathrm{v}$ increase approximately at the same percentage in all figures of interest. Figure (9) shows the variation of the total energy which is called the vibrational - rotational energy at $\mathrm{v}=0$ as a function of rotational quantum number. It can be shown that the variation is as in figure (3) which means that the vibrational energy has predominate values in comparison with the rotational energy.

The main conclusion to be drawn from all the results that it can be shown that the values for DF - and $\mathrm{HCl}$ - lasers are identical. It allows performing a comparison of their characteristics. Because of the larger number of vibrational-rotational transitions that lase in DF - lasers, power is distributed over a larger number of states than in HF. Most of the computer modeling was available for the HF - laser, since the more reliable rate constants of chemical reactions and of processes of vibrational - rotational translational kinetics for the mixture $\mathrm{H}_{2}+\mathrm{F}_{2}$ are known in comparison with the mixture $\mathrm{D}_{2}+\mathrm{F}_{2}$. Ways of achieving extreme energy characteristics of $\mathrm{HF} / \mathrm{DF}$ and $\mathrm{HCl} / \mathrm{DCl}$ 
lasers were determined as a results of theoretical investigations of vibrational - rotational parameters. The close values for both $\mathrm{DF}$ and $\mathrm{HCl}$ lasers constants give nearly the same results of calculations.

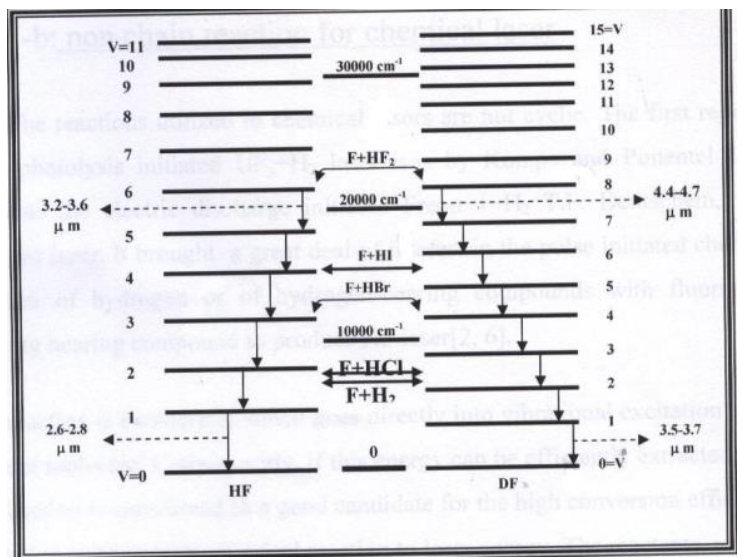

Figure (1) The HF and DF vibrational energy levels.

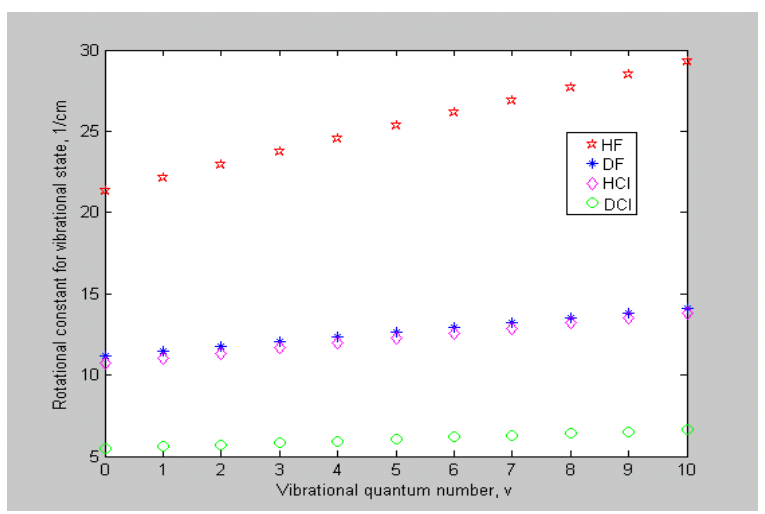

Figure (2) Rotational constant for vibrational state as a function vibrational quantum number.

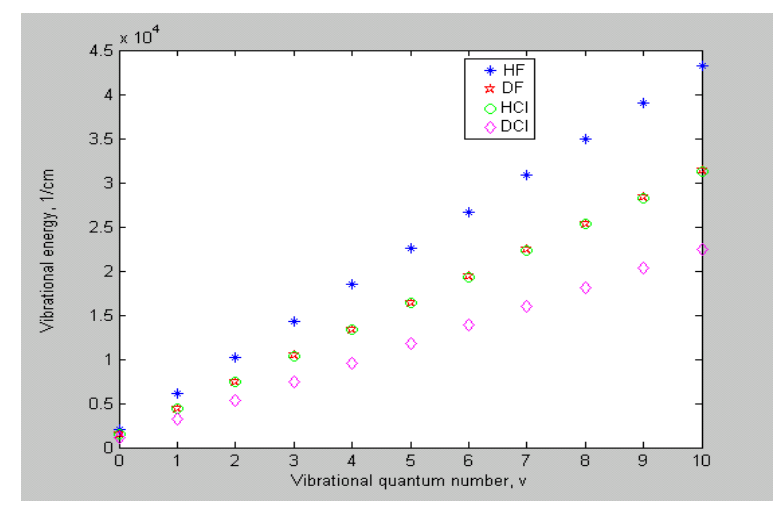

Figure (3) vibrational energy as a function vibrational quantum number.

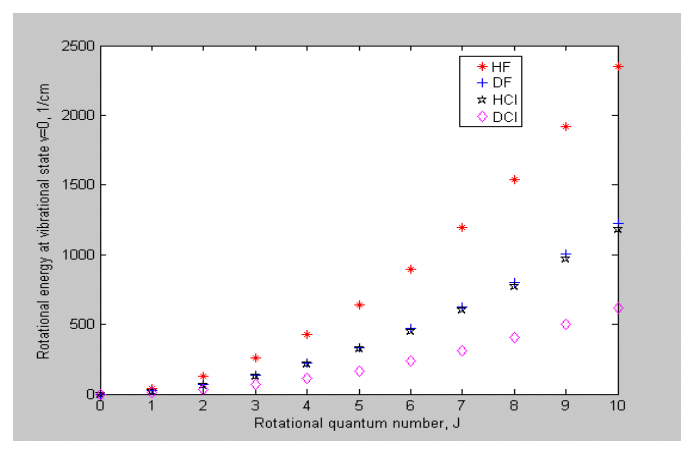

Figure (4) Rotational energy at vibrational state $v=0$ as a function rotational quantum number.

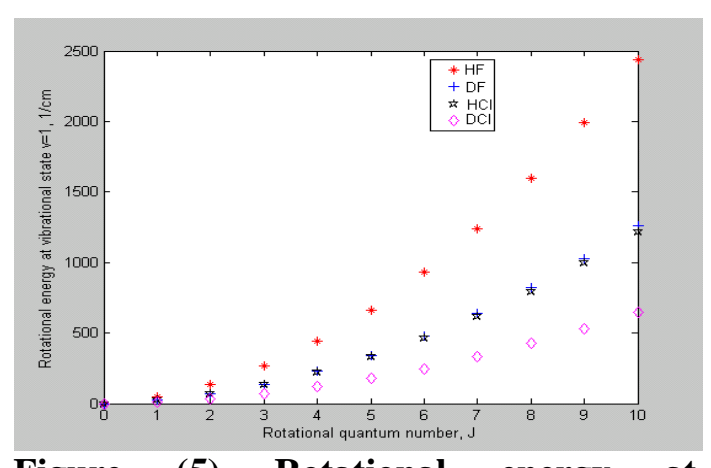

Figure (5) Rotational energy at vibrational state $v=1$ as a function rotational quantum number.

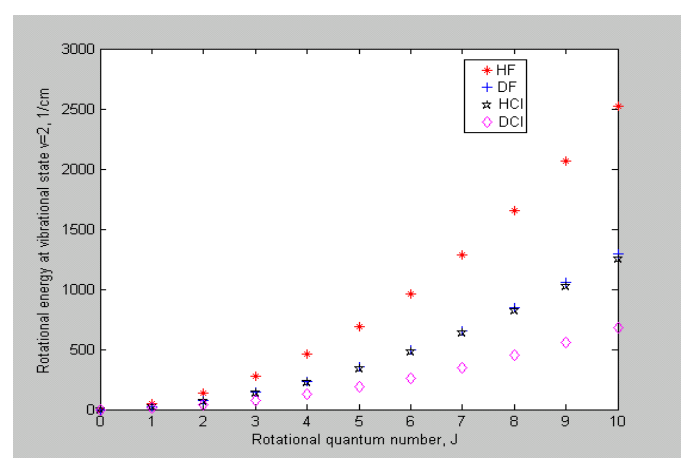

Figure (6) Rotational energy at vibrational state $v=2$ as a function rotational quantum number. 


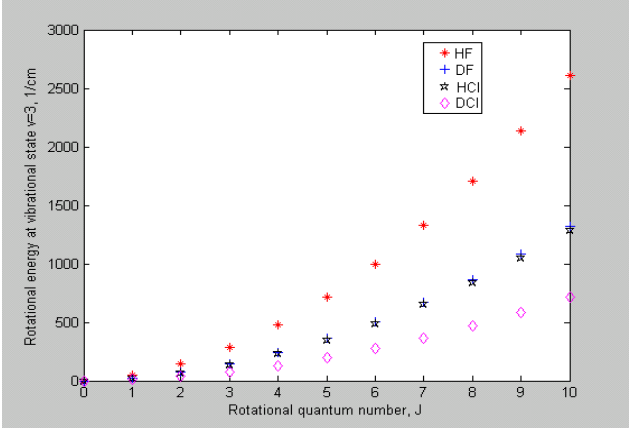

Figure (7) Rotational energy at vibrational state $v=3$ as a function rotational quantum number.

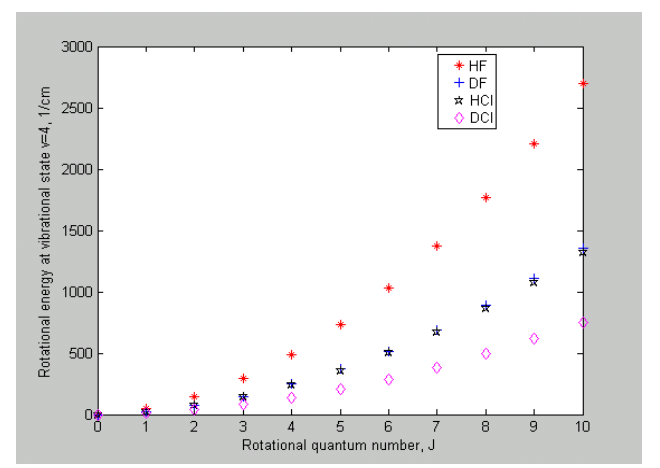

Figure (8) Rotational energy at vibrational state $v=4$ as a function rotational quantum number.

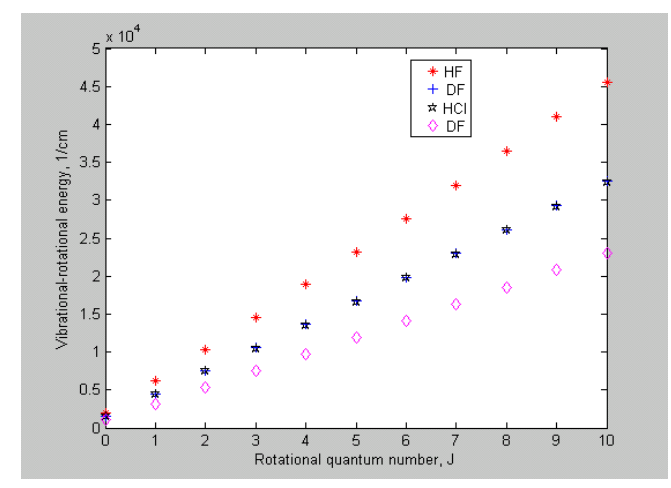

Figure (9) Vibrational - rotational as a function of rotational quantum number at a vibrational state $v=0$.

\section{REFERENCES:}

1. Azarov, M. A., Aleksandrov, B. S., Drozdov, V. A., and Troshchinenko,G. A., (2000): Influence of the caity losses on the energy and spectral characteristics of a plused chemical chain - reaction $\mathrm{HF}(\mathrm{DF})$ laser, Quantum Electronic 30(1) : 30-36.

2. Basov, N. G., Bashkin, A. S., Igoshin, V. L., Oraevsky, A. N., and Shcheglov, V. A., (1990) : Chemical Laser, (Springer Verlag) : 33-34.

3. Bravy, B.G., Vasiliev, G.K., Kiriyanov, V.L., Makarov, E.F., and Chernyshev, Yu.A., (1996): Pulsed Chemical Hydrogen Fluoride Laser Problems, Developments, and Applications, BRAS Physics/Supplement Phys. Vibrat. 60:117.

4. Das, P., (1991): Laser and Optical Engineering, (Springer Verlag).

5. Detweiler, G. L., Sentman, L. H., and Carroll, D. L., (2005) : The Possibility of Hot Reaction Enhancement of $\mathrm{CW}$ HF Laser Performance, $\quad 36^{\text {th }} \quad$ AIAA Plasmsdynamics and Laser Conference : 1-14.

6. Deutsch, T. F., (1967): Molecular laser action in hydrogen and deuterium halides, Appl. Phys. Lett., 10 : 234-236.

7. Eletskii, A.V.,(1981): Processes in Chemical Laser, Sov. Phys. Usp., 24:475.

8.Gross, R.W.F., and Bott, J. F., (1976) : Handbook of chemical laser, (Johen Wiley, New York).

9.Igoshin, V. I., Pichugin, S. Yu., (1992) : Theoretical simulation of lasers utilizing vibrational $\quad-$ rotational transitions in diatomic molecules allowing for anharmonicity and rotational equilibrium, Sov. J. Quantum electron, 22 : 337-340.

10. Ilyushin, A. A., (1998) : Numerical Modeling of Two-Dimensional Flow and Kinetic Processes in a Chemical HF CW Laser, Applied Energy: Russian Journal of Fuel, Power and Heat Systems, 36 : $61-67$.

11. Kondratiev, V. N., (1969) :The theory of kinetics, In comprehensive chemical kinetics, by Bamford, C. H., and Tipper, C. F. H., Volume 2. 
12. Nichols, D. B., Hall, R. B., McClure, J. D., (1976): Photoinitiated $\mathrm{F}_{2}+\mathrm{H}_{2} /$ $\mathrm{D}_{2}$ chain - reaction laser with high electrical efficiency, J. Appl.. Phys. 47: 4026.

13. Palekar, A., (2004): Hydrodynamics Inside A Chemical Laser, Internet :1-15. 14. Park, J. S. and Baek, S. W.,(2005): Effects of pressure ratio on population inversion in a DF chemical laser with concurrent lasing, J. Quant. Spect. Radia. Trans.,92 : 31-49.

15. Perram, G. P., (2002): Chemical Lasers, Internet : 1 -36.

16. Stitch M.L., (1979): Laser Handbook, (North-Holland,

\section{دراسة نظرية للطاقة الدورانية ـ الاهتزازية لبعض جزيئات هاليدات الهيدروجين}

\section{رفاه عبد الهادي}

*جامعة بغداد/ كلية العلوم للبنات / قسم الفيزياء.

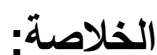

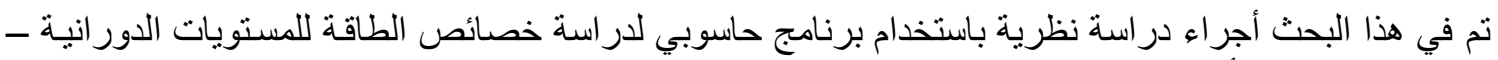

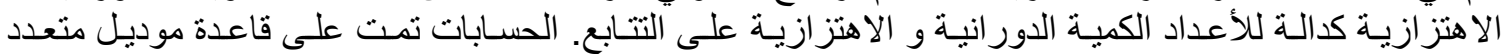

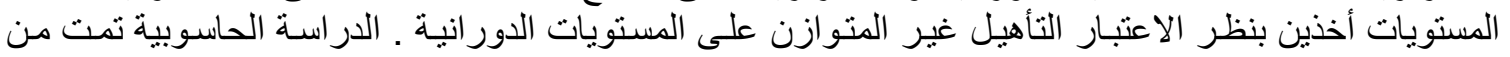

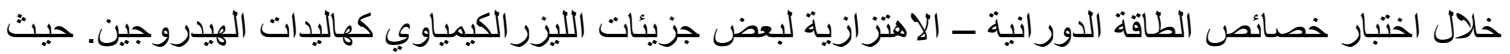

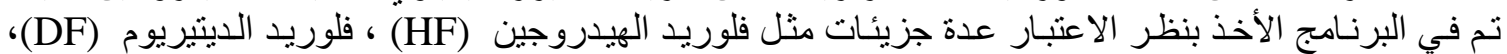

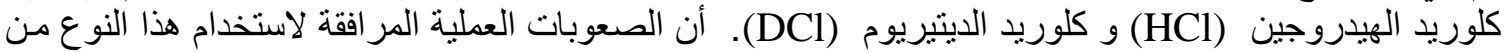

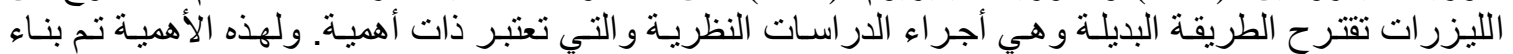

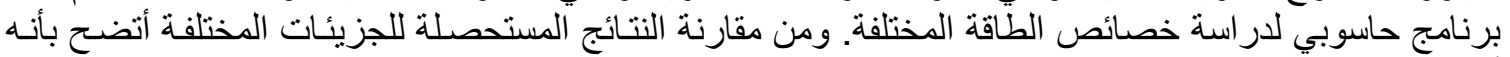

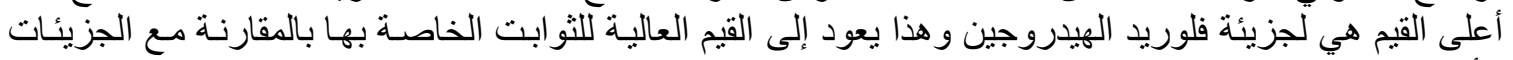

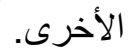

